\title{
Longitudinal patterns of cancer patient reported outcomes in end of life care predict survival
}

\author{
George J. Stukenborg ${ }^{1}$ - Leslie J. Blackhall ${ }^{2}$. James H. Harrison ${ }^{1}$ • Patrick M. Dillon ${ }^{2}$. \\ Paul W. Read ${ }^{3}$
}

Received: 31 July 2015 / Accepted: 8 November 2015 / Published online: 16 November 2015

(C) The Author(s) 2015. This article is published with open access at Springerlink.com

\begin{abstract}
Purpose Patients with advanced cancer typically demonstrate sharp deterioration in physical function and psychological status during the last months of life. This study evaluates the relationship between survival in patients with advanced cancer and longitudinal assessment of anxiety, depression, fatigue, pain interference, and/or physical function using the US National Institute of Health Patient Reported Outcomes Information System.

Methods Mixed-effects models were used to evaluate patientreported outcome trajectories over time among patients with advanced loco-regional or metastatic cancer receiving care in a hospital-based palliative care clinic. Cox regression analysis was used to assess the statistical significance of differences in the probability of survival associated with patient-reported outcome scores.

Results A total of 472 patients completed 1992 assessments during the 18-month study period. Longitudinal scores for fatigue, pain interference, and physical function demonstrated statistically significant non-linear trajectories. Scores for depression, fatigue, pain interference, and physical function were highly statistically significant predictors of survival $(p<0.01)$. Clinically meaningful differences in the probability
\end{abstract}

George J. Stukenborg

gstukenborg@virginia.edu

1 Department of Public Health Sciences, University of Virginia School of Medicine, P.O. Box 800717, Charlottesville, VA 22908-0821, USA

2 Department of Medicine, University of Virginia School of Medicine, P.O. Box 800717, Charlottesville, VA 22908-0821, USA

3 Department of Radiation Oncology, University of Virginia School of Medicine, P.O. Box 800717, Charlottesville, VA 22908-0821, USA of survival were demonstrated between patients with scores at the 25 th vs. 75 th percentiles, with absolute differences in survival at 6 and 12 months after assessment from 10 to 18 percentage points.

Conclusions Patient-reported outcomes can be used to reliably estimate where patients are along the trajectory of deteriorating health status leading toward the end of life, and for identifying patients with declining symptoms in need of referral to palliative care or more aggressive symptom management.

Keywords Cancer $\cdot$ EHR $\cdot$ PROMIS

\section{Introduction}

Patients with advanced cancer have an illness trajectory that typically begins with a period of relatively preserved functional status followed by a period of marked deterioration and increased symptoms during the last months of life [1-3]. This period of functional decline is associated with frequent hospitalization and increased costs of care. Analysis of Medicare data for patients who died with poor-prognosis cancers indicates that $65 \%$ were hospitalized and $25 \%$ had intensive care unit stays within 30 days of their death [4].

Early identification of patients who are approaching the end of life can improve patient outcomes by improving clinical decision-making. Earlier referral to hospice and palliative care can result in improvements in quality of life and even length of life [5-7]. Aggressive anticancer treatment in the terminal phase increases costs of care, may reduce quality of life, and may fail to yield any improvement in survival [8]. Between 20 and $50 \%$ of patients with advanced cancer receive chemotherapy within 30 days of death [9]. Patients with systemic chemotherapy in the last months of life have a 
substantially increased risk of dying in an intensive care unit [10]. Chemotherapy in the last weeks of life is also associated with reduced likelihood of receiving hospice care [11].

Patient self-reported health status measures are useful for estimating survival in cancer patients and can provide unique information of importance for monitoring patient health status trajectories [12-15]. While numerous patient-reported outcome measures have been developed over the years, recent national efforts have produced standardized assessment measures. Beginning in 2004, the National Institutes of Health sponsored the development of the Patient-Reported Outcomes Measurement Information System (PROMIS) to provide a uniform methodology for quantifying physical, mental, and social health across patient populations [16, 17]. The PROMIS project measures provide reliable and valid assessment of patient self-reported health status for use in cancer patient care delivery and clinical research $[18,19]$. This analysis examines the relationship between survival and PROMIS domain measures among patients with advanced cancer at a large health system and provides evidence regarding their potential utility in identifying those with declining symptoms in need of referral to palliative care or more aggressive symptom management.

\section{Methods}

Computer-based assessment of patient-reported outcomes (PROs) was implemented for patients with advanced locoregional or metastatic cancer (stage 4 solid tumor or other advanced cancer identified as incurable by the referring oncologist or surgeon) who were referred to this program at the University of Virginia Health System Cancer Center. Palliative care services include an outpatient clinic located in the cancer center, inpatient consultation, a palliative care unit, and home hospice components. Patients referred to this clinic are typically followed concurrently with medical oncology, radiation oncology, and surgical oncology for symptom management and end of life care planning. The palliative care clinic staff includes RNs, MDs and NPs, with available social worker and chaplaincy services.

Assessment was initiated for all patients beginning in October 2013, following a pilot testing phase completed in February 2013 [20]. The set of PRO measures collected for each patient was selected based on national recommendations and physician and patient stakeholder review [21]. The assessment includes five PROMIS health domains: anxiety, depression, fatigue, pain interference, and physical function. Additional PROs were developed for the project to address the following cancer-specific symptoms: bowel function, nausea, vomiting, anorexia, dyspnea, and peripheral neuropathy.

Patient data available from the health system Electronic Health Record (EHR) and from health system administrative records was collected to identify patient demographics, cancer diagnoses, and survival outcomes for all patients with assessments. Cancer diagnoses were identified using ICD-9-CM diagnosis codes reported in hospital and outpatient care encounter summaries for events occurring up to 2 years prior to the date of the first assessment. Individual ICD-9-CM codes were grouped into categories of site-specific cancer diagnoses using the criteria of the Agency for Healthcare Research and Quality Clinical Classification System [22].

The collection of PROs was implemented using software integrated within the patient's electronic health record. The assessment software was accessed by patients through an online browser using a tablet computer. The assessment protocol included instructions on the assessment process and on the use of the tablet computer, which was provided by the clinic staff to patients at each visit. Direct assistance with the assessment process was provided by clinical staff to any patient who requested assistance. Patients completed an assessment at each routine follow-up appointment, before their clinical encounter. The availability of direct assistance with the assessment was included in the protocol based on the results of the pilot study, which demonstrated that while most patients were able to complete the assessment without assistance, some patients required direct assistance with the use of the tablet computer, with the assessment software, or with the assessment questions [20].

Completed assessments were posted immediately to the patients' EHR records. Displays of both current and longitudinal results were available during the patient encounter for use in therapeutic decision-making, assessment of response to treatment, symptom management, and end of life care planning. This analysis includes results collected for the five PROMIS health domains during the 18-month period from October 2013 through March 2015.

Each PROMIS domain was scored using the version 1.0 short form item series, with 5 ordinal response options (1 to 5) for each question in the domain [23]. Mean scores for the total responses indicate whether patients have more or less of the domain being measured. For example, higher mean scores indicate worse anxiety, depression, fatigue, and pain interference, but better physical function. Mean scores were calculated for each domain, for each patient assessment. Mean scores were also calibrated to the weighted distribution of mean scores from a large representative sample of the US general population, using the "T-score" algorithm [24]. Populationcalibrated scores are referent to the surveyed population mean of 50 and standard deviation of 10 , such that a patient with a Tscore of 40 in the study population has a score that is one standard deviation below the US general population mean. Frequency distributions for PROMIS domain score values were calculated at the mean score scale and T-score scale.

Patients in the study population were referred at different points in time in the progression of their disease. 
Given this heterogeneity in stage of disease progression among patients, there was no shared reference point for defining the start point of trajectory patterns measured prospectively in the total study population. Rather, trajectory patterns were assessed retrospectively for deceased patients only, using the patient's date of death as a shared reference point for the end of the trajectory.

Multi-level mixed-effects models were used to estimate the significance and direction of PROMIS domain score trajectories over time. Differences among patients in the number of assessments and in the spacing between assessments prior to death are accommodated by the hierarchical structure of these models [25]. The shape of the score trajectories was estimated using linear, quadratic, and spline functions. Linear models estimate only the linear association between scores and months prior to death. Quadratic models have components for estimating both the linear and non-linear association. Spline functions are piecewise polynomials with components that combine into curved functions fit to the pattern of scores. Spline functions yield maximum precision for estimation of nonlinear relationships [26]. The statistical significance of each functional relationship was assessed using the fixed effect F-test statistic $p$ value calculated for each model parameter. The extent of non-linearity in the relationship between PROMIS scores and months prior to death was measured using the Akaike information criterion (AIC) statistic to calculate the probability of information loss from excluding non-linear relationships in the models [27].

Cox regression analysis was used to assess the statistical significance of differences in the probability of survival by months post assessment associated with PROMIS domain scores for all patients in the study population. The statistical significance of the model score coefficients was assessed using the Wald chi-square test statistic. Survival probabilities at the time of assessment were estimated using models with time-dependent covariates conditional on the interaction between the PROMIS domain score and the number of months elapsed since the beginning of follow-up [28-30]. A logarithmic function was used to represent the non-linear shape of change in scores over time [31]. Probabilities of survival at 6 and 12 months post assessment were estimated with reference to the 25th, 50th, and 75th percentiles of the domain score distributions. The statistical significance of the difference in survival by quartiles of the PROMIS domain scores was calculated using the stratified log-rank test statistic.

Data management, statistical programming, and graphics programming was conducted using SAS 9.4 and R statistical software, version 2.13 (R foundation for Statistical Computing, Vienna, Austria). The statistical significance of parameter effect estimates and of test statistics was assessed at the a priori threshold value of $p<0.01$.
Table 1 Study population demographics and cancer diagnosis groups

\begin{tabular}{|c|c|c|}
\hline & Number & Percent \\
\hline Total & 472 & 100.00 \\
\hline Deceased & 203 & 43.01 \\
\hline \multicolumn{3}{|l|}{ Race } \\
\hline Asian & 2 & 0.42 \\
\hline Black or African American & 64 & 13.56 \\
\hline Other/unspecified & 18 & 3.81 \\
\hline White & 388 & 82.20 \\
\hline \multicolumn{3}{|l|}{ Ethnicity } \\
\hline Hispanic & 7 & 1.48 \\
\hline Non-Hispanic & 465 & 98.52 \\
\hline \multicolumn{3}{|l|}{ Sex } \\
\hline Female & 267 & 56.57 \\
\hline Male & 205 & 43.43 \\
\hline \multicolumn{3}{|l|}{ Health Insurance } \\
\hline Medicaid & 70 & 14.83 \\
\hline Medicare & 173 & 36.65 \\
\hline Private/commercial health insurance & 169 & 35.81 \\
\hline Self-pay & 20 & 4.24 \\
\hline TRICARE (US military) & 3 & 0.64 \\
\hline Uninsured & 18 & 3.81 \\
\hline \multirow[t]{2}{*}{ Unknown } & 19 & 4.01 \\
\hline & Mean & Std dev \\
\hline Age in years at referral & 58.9 & 12.15 \\
\hline \multicolumn{3}{|l|}{ Site-specific cancer diagnosis groups } \\
\hline Bronchus, lung & 82 & 17.37 \\
\hline Breast & 69 & 14.62 \\
\hline Head and neck & 56 & 11.86 \\
\hline Ovary & 43 & 9.11 \\
\hline Other GI organs; peritoneum & 42 & 8.90 \\
\hline Colon & 40 & 8.47 \\
\hline Other non-epithelial cancer of skin & 40 & 8.47 \\
\hline Bone and connective tissue & 35 & 7.42 \\
\hline Brain and nervous system & 33 & 6.99 \\
\hline Uterus & 30 & 6.36 \\
\hline Other female genital organs & 29 & 6.14 \\
\hline Rectum and anus & 28 & 5.93 \\
\hline Melanomas of skin & 27 & 5.72 \\
\hline Cervix & 26 & 5.51 \\
\hline Prostate & 23 & 4.87 \\
\hline Liver and intrahepatic bile duct & 22 & 4.66 \\
\hline Kidney and renal pelvis & 21 & 4.45 \\
\hline Pancreas & 20 & 4.24 \\
\hline Benign neoplasm of uterus & 19 & 4.03 \\
\hline Non-Hodgkin's lymphoma & 15 & 3.18 \\
\hline Bladder & 11 & 2.33 \\
\hline Other respiratory and intra-thoracic & 11 & 2.33 \\
\hline Thyroid & 11 & 2.33 \\
\hline Stomach & 11 & 2.33 \\
\hline Esophagus & 10 & 2.12 \\
\hline Leukemias & 5 & 1.06 \\
\hline Multiple myeloma & 5 & 1.06 \\
\hline Hodgkin's disease & 2 & 0.42 \\
\hline
\end{tabular}




\section{Results}

All patients presenting in the clinic who met the selection criteria were included in the study, and all selected patients were able to complete the assessments. A total of 472 patients completed assessments during the 18-month study period, and 203 of these patients (43\%) died during the study period. Demographic and diagnostic characteristics of the study population are listed in Table 1 . The mean age was 58.9 years, and $56.6 \%$ of patients were female. Cancer of the bronchus and lung (17.4\%), breast $(14.6 \%)$, head and neck (11.9\%), ovary $(9.1 \%)$, colon $(8.5 \%)$, and other cancers for gastro-intestinal sites and peritoneum $(8.9 \%)$ were the most frequently occurring cancer sites reported for the study population.

A total of 1992 assessments were completed during the study period, with repeated assessments available for most patients: $>1$ (73.3\%), >2 (55.1\%), >3 (41.5\%). The mean number of assessments per patient was 4.2 , with a mean of 3.8 months between assessments. Figure 1 illustrates the patterns of consecutive scores reported for three example patients who died during the study period.

Table 2 lists the distributional characteristics of the PROMIS mean domain scores and the T-score scaled mean domain scores in the study population. The T- score scaled results demonstrate that patients in the study population had overall score means that were higher than the US general population medians for anxiety (56.8), depression (51.9), fatigue (56.0), and pain interference (58.1) and had lower mean scores for physical function (44.4).

Table 2 also displays the multi-level mixed-effects model analysis results for functional trends in scores during the months prior to death. Statistically significant linear relationships were demonstrated for fatigue, pain interference, and physical function scores. Linear trends in anxiety and depression scores were not statistically significant. Statistically significant non-linear trends were demonstrated by the quadratic model for fatigue, pain interference, and physical function scores. Quadratic model terms were not significant for trends in anxiety scores $(p=0.4207, p=0.5055)$ and were near significance for trends in depression scores $(p=0.0599$, $p=0.0545)$ over the months preceding death. The spline models demonstrate that non-linear function components were significant or nearly significant for each of the five PROMIS score domains. Figure 2 illustrates the shapes of the estimated spline model trajectories for each PROMIS score domain among the deceased patients. Non-linear functional transformations using the
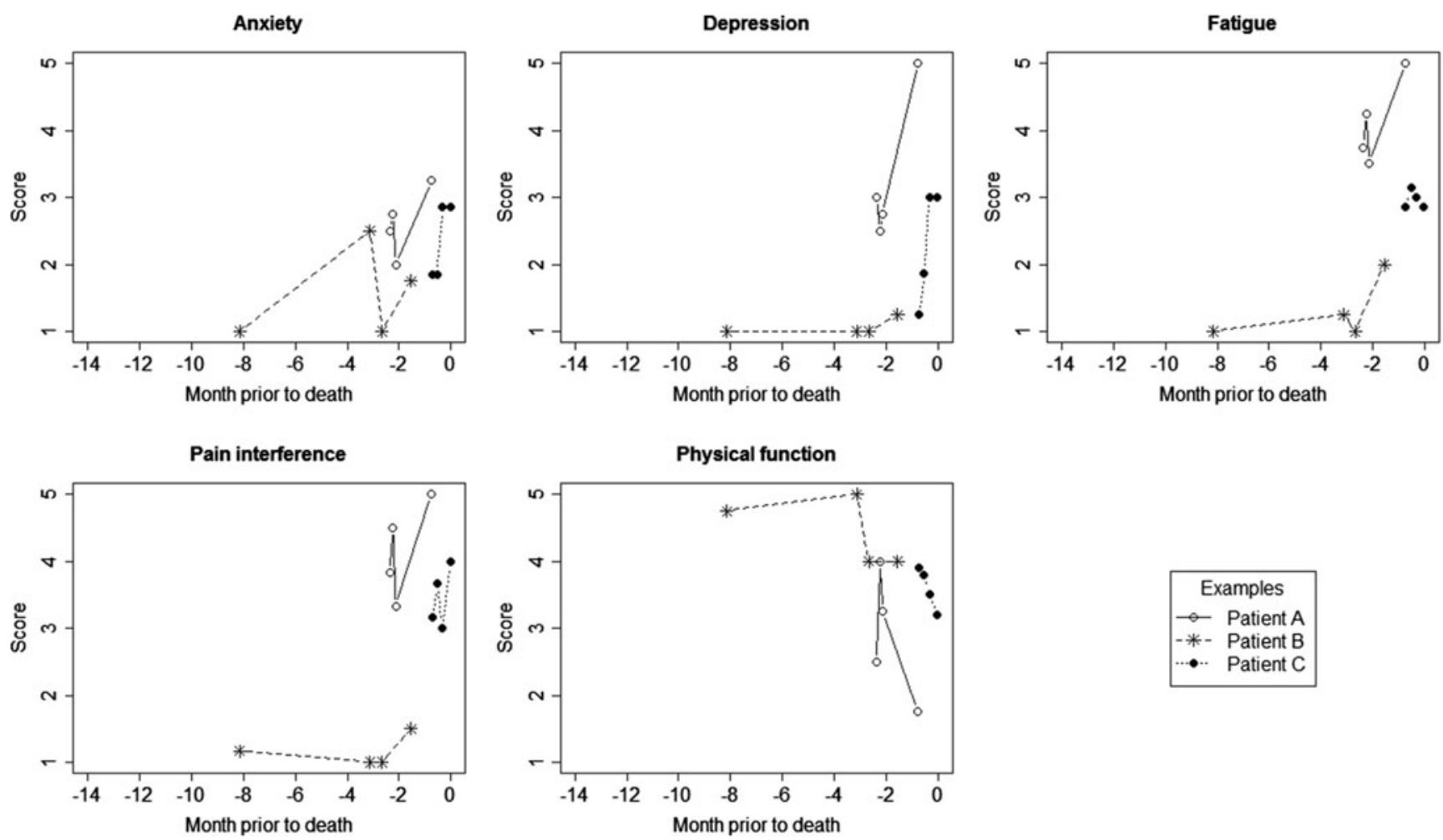

Fig. 1 A plot of example assessment score values reported for three patients who died during the study period, for each PROMIS domain. Dates of assessment are indexed by months prior to death along the horizontal access, and mean domain score values reported for each

assessment are represented on the vertical axis. Consecutive scores for example patients are linked by reference lines, for each of the three example patients identified in the plot legend 
Table 2 PROMIS score distributions and statistical model results

\begin{tabular}{|c|c|c|c|c|c|}
\hline & Anxiety & Depression & Fatigue & Pain interference & Physical function \\
\hline Mean score scale mean & 2.46 & 1.95 & 2.84 & 2.67 & 3.93 \\
\hline Mean score scale 25 th percentile & 1.75 & 1.00 & 2.25 & 1.66 & 3.30 \\
\hline Mean score scale 50 th percentile & 2.50 & 1.75 & 2.75 & 2.66 & 4.00 \\
\hline Mean score scale 75 th percentile & 3.00 & 2.50 & 3.50 & 3.66 & 4.75 \\
\hline T-score scale mean & 56.80 & 51.94 & 55.96 & 58.11 & 44.40 \\
\hline T-score scale 25 th percentile & 52.10 & 38.20 & 51.50 & 53.20 & 38.90 \\
\hline T-score scale 50 th percentile & 58.40 & 53.20 & 55.60 & 59.50 & 43.10 \\
\hline T-score scale 75 th percentile & 62.50 & 58.20 & 61.30 & 64.80 & 49.60 \\
\hline \multicolumn{6}{|l|}{ Multi-level mixed-effects models (deceased patients only) } \\
\hline \multicolumn{6}{|l|}{ Linear model } \\
\hline Linear term $p$ value & 0.6367 & 0.8048 & 0.0001 & 0.0005 & $<0.0001$ \\
\hline \multicolumn{6}{|l|}{ Quadratic model } \\
\hline Linear term $p$ value & 0.4207 & 0.0599 & $<0.0001$ & $<0.0001$ & $<0.0001$ \\
\hline Quadratic term $p$ value & 0.5055 & 0.0545 & 0.0038 & 0.0018 & $<0.0001$ \\
\hline \multicolumn{6}{|l|}{ Spline model } \\
\hline Linear term $p$ value & 0.2301 & 0.3679 & 0.4053 & 0.6804 & 0.1371 \\
\hline Spline term part $1 p$ value & 0.0393 & 0.0236 & 0.3653 & 0.5419 & 0.0593 \\
\hline Spline term part $2 p$ value & 0.0061 & 0.0008 & 0.0175 & 0.0205 & 0.4479 \\
\hline Probability of information loss by excluding non-linear terms & 0.1353 & 1.0000 & 1.0000 & 1.0000 & 1.0000 \\
\hline \multicolumn{6}{|l|}{ Cox regression models (all patients) } \\
\hline Score term $p$ value & 0.8692 & 0.0005 & $<0.0001$ & $<0.0001$ & $<0.0001$ \\
\hline Survival curve homogeneity over score quartiles log-rank $p$ value & 0.1033 & 0.0189 & 0.0031 & $<0.0001$ & $<0.0001$ \\
\hline \multicolumn{6}{|l|}{ Probabilities of survival } \\
\hline 25 th percentile at 6 months & 0.95 & 0.96 & 0.98 & 0.98 & 0.89 \\
\hline 50 th percentile at 6 months & 0.91 & 0.93 & 0.95 & 0.94 & 0.96 \\
\hline 75th percentile at 6 months & 0.94 & 0.92 & 0.91 & 0.90 & 0.99 \\
\hline 25 th percentile at 12 months & 0.80 & 0.82 & 0.87 & 0.87 & 0.72 \\
\hline 50th percentile at 12 months & 0.77 & 0.79 & 0.82 & 0.80 & 0.83 \\
\hline 75th percentile at 12 months & 0.79 & 0.76 & 0.73 & 0.73 & 0.89 \\
\hline
\end{tabular}

spline models significantly improved the fit of trajectories estimated for the depression, fatigue, pain interference, and physical function scores. The probability of information loss associated with excluding non-linear terms was 1.0 for each of these four PROMIS domains.

Figure 3 presents plots of the exact functional relationships over time for scores at the 25th, 50th, and 75 th percentile reference values of each of the five PROMIS domain score distributions. Reference values for the mean score and T-score values corresponding to the 25th, 50th, and 75th percentiles of each PROMIS domain score distribution are listed in Table 2. Results for the stratified log-rank test statistic demonstrate that the differences in survival functions by quartiles of the anxiety and depression scores were not statistically significant. However, highly statistically significant differences in survival were demonstrated for quartiles of scores for fatigue, pain interference, and physical function $(p<0.01)$.

The predictive relationship of PROMIS domain scores to post assessment survival was evaluated for all patient assessments. Table 2 lists results obtained from the Cox regression analysis. Each of the PROMIS domain scores had highly statistically significant relationships to patient survival, except the PROMIS domain scores for anxiety $(p=0.8692)$. Large absolute differences in survival at 6 and 12 months post assessment occur between patients with domain scores at the 25th and 75th percentiles for fatigue, pain interference, and physical function. Patients who reported high levels of physical function (scores at 75th percentile) had a 0.99 probability of 6-month survival and a 0.89 probability of 12-month survival. In comparison, patients with low levels of physical function (25th percentile) had a 

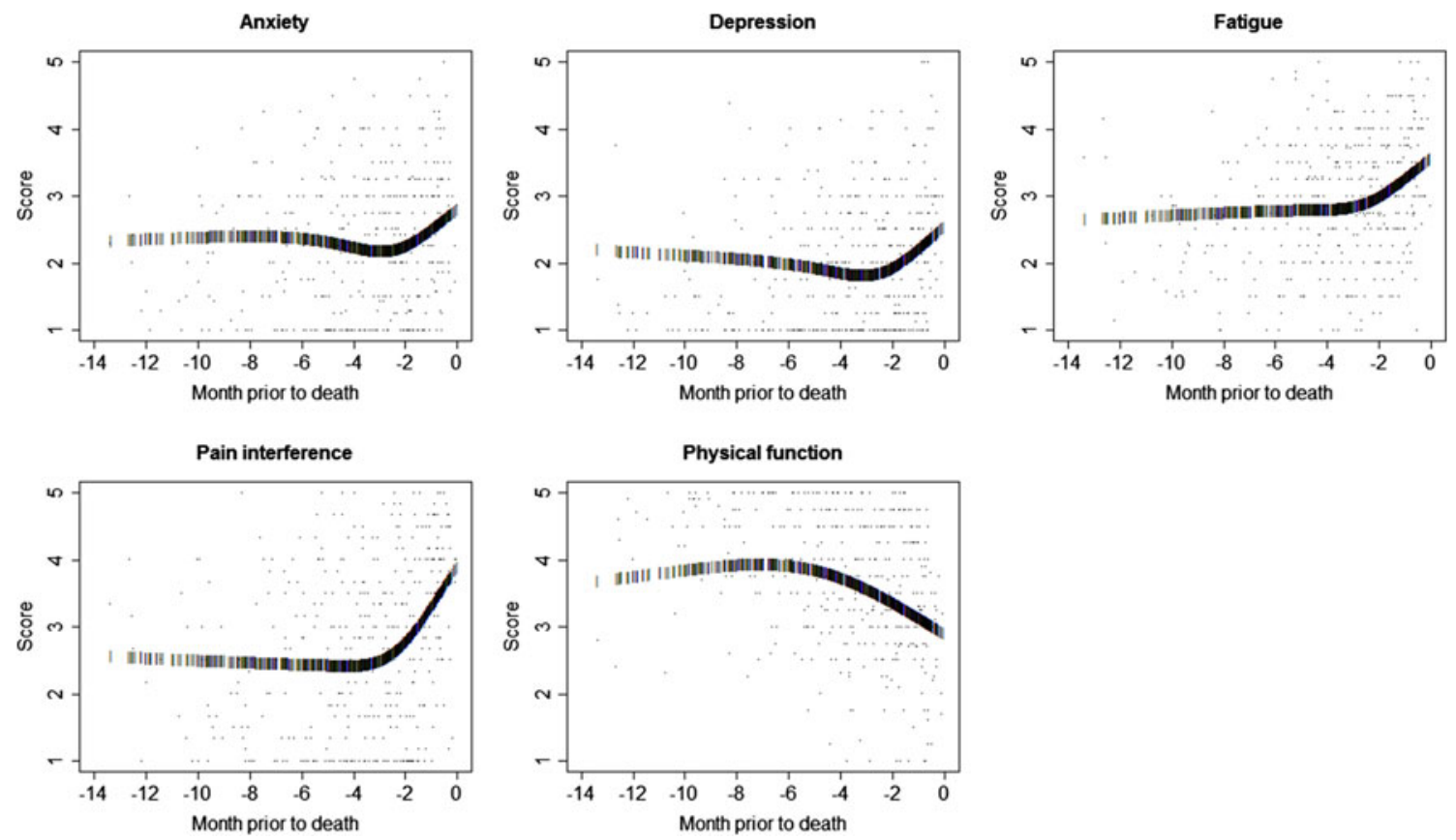

Fig. 2 A plot of the spline function trajectory of estimated score values reported for deceased patients, for each PROMIS domain. All mean score values are plotted, with dates of assessment indexed by months prior to death along the horizontal access, and mean domain score values

0.89 probability of 6 -month survival $(-0.10$ point difference) and a 0.72 probability of 12-month survival represented on the vertical axis, for each PROMIS domain. The spline function trajectory of estimated score values is depicted by bars plotted for each assessment date, superimposed on the scatterplot of all score values

( -0.17 point difference). Table 2 lists the absolute differences in 6- and 12-month survival for patients at the
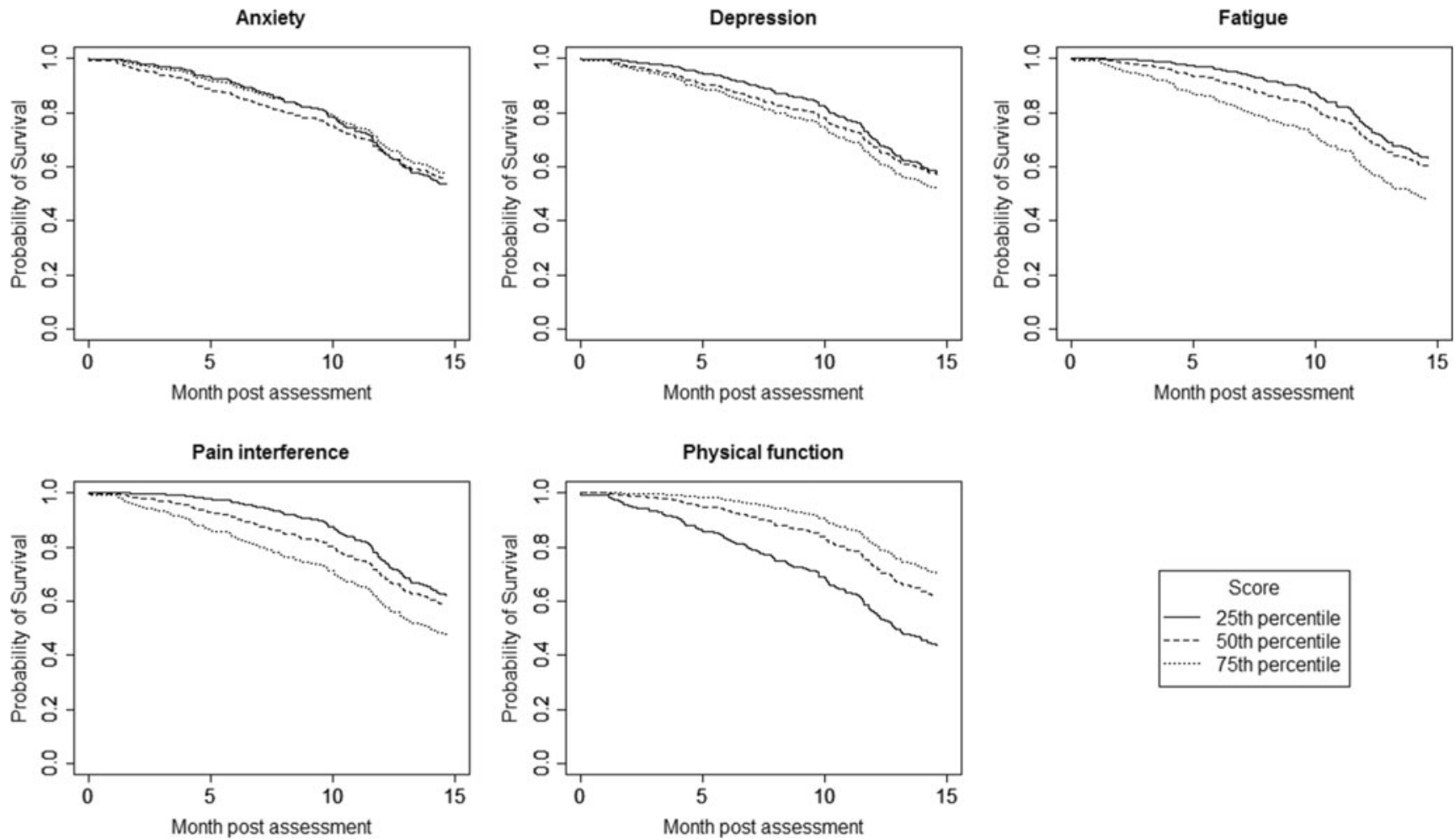

Fig. 3 A plot of the survival functions estimated for all patients in the study population, with reference to the mean scores at the 25 th, 50th, and domain. Months post assessment are indexed on the horizontal access. Estimated probabilities of survival are indexed on the vertical axis 
25th and 75th percentiles of each of the five PROMIS domain scores.

\section{Discussion}

This research demonstrates that PROMIS scores for fatigue, pain interference, and physical function provide reliable indications of reduced 6- and 12-month survival in patients with advanced cancer. In our study population, patients with scores at the high or low ends of the scales demonstrate clinically meaningful absolute differences in the probability of survival at 6 and 12 months after assessment.

The PROMIS assessment tools provide a standardized and readily available method for scoring fatigue, pain interference, and physical function. Regular computer-based patient assessment provides longitudinal information useful for informing clinical decisions during patient visits, while ensuring that patient data is fully protected within the EHR. When PROs are available in routine EHR reports, patients with severe symptoms or trend patterns of concern can be identified early and referred to appropriate services prior to symptom escalation to the point of requiring inpatient management.

Patient-reported outcomes are increasingly recognized as relevant to oncology. Patient-reported outcomes provide a unique patient perspective on treatment benefit and risk that goes beyond clinician-reported adverse events [32]. Patients and clinicians often differ in their assessment of the severity and impact of cancer and treatment-related symptoms [33]. As a result, PROs can provide crucial information for guiding patient care. The Center for Medicare and Medicaid Innovation is developing new payment and delivery models for oncology care that utilize patient-reported outcomes assessment and recommends the use of the PROMIS short forms for this purpose [34].

In the era of increasingly shared care and shortened patient visits, computer-based assessment of PROs can enhance the continuity of care provided. Clinicians often must make decisions using information from a snapshot in time, and without the patient's longitudinal history being available and readily accessible at the point of care, it can be difficult to identify patients with meaningful changes in these status measures. Longitudinal PROs in the EHR are highly useful for identifying patients with reduced probabilities of survival, and for identifying patients with declining symptoms in need of referral to palliative care or more aggressive symptom management. We hypothesize that end of life health interventions that target reducing fatigue and pain interference and increasing physical function are the ones most likely to improve quality of life and prolong survival.

Acknowledgments The project described is supported by Grant Number 1C1CMS331031-01-00 from the Department of Health and Human Services, Centers for Medicare \& Medicaid Services (PI Paul Read, MD). The contents of this publication are solely the responsibility of the authors and do not necessarily represent the official views of the Department of Health and Human Services or any of its agencies. PROMIS ${ }^{\circledR}$ was funded with cooperative agreements from the National Institutes of Health $(\mathrm{NIH})$ Common Fund Initiative (U54AR057951, U01AR052177, U54AR057943, U54AR057926, U01AR057948, U01AR052170, U01AR057954, U01AR052171, U01AR052181, U01AR057956, U01AR052158, U01AR057929, U01AR057936, U01AR052155, U01AR057971, U01AR057940, U01AR057967, U01AR052186). The contents of this article uses data developed under PROMIS. These contents do not necessarily represent an endorsement by the US Federal Government or PROMIS. See www.nihpromis.org for additional information on the PROMIS initiative.

\section{Compliance with ethical standards}

Conflict of interest Dr. Stukenborg has full access to all of the data in the study, conducted the data analysis, and takes responsibility for the integrity of the data and the accuracy of the data analysis. Dr. Stukenborg has full control of the data and agrees to allow the journal to review the data if requested.

Grant support The project described is supported by Grant Number 1C1CMS331031-01-00 from the Department of Health and $\mathrm{Hu}$ man Services, Centers for Medicare \& Medicaid Services (PI Paul Read, MD). The contents of this publication are solely the responsibility of the authors and do not necessarily represent the official views of the Department of Health and Human Services or any of its agencies.

Funding PROMIS ${ }^{\circledR}$ was funded with cooperative agreements from the National Institutes of Health (NIH) Common Fund Initiative (U54AR057951, U01AR052177, U54AR057943, U54AR057926, U01AR057948, U01AR052170, U01AR057954, U01AR052171, U01AR052181, U01AR057956, U01AR052158, U01AR057929, U01AR057936, U01AR052155, U01AR057971, U01AR057940, U01AR057967, U01AR052186). The contents of this article uses data developed under PROMIS. These contents do not necessarily represent an endorsement by the US Federal Government or PROMIS. See www.nihpromis.org for additional information on the PROMIS initiative.

Sources of supports The sources of financial and material support had no role in the design or conduct of the study; collection, management, analysis, or interpretation of the data; or preparation, review, or approval of the manuscript.

Disclosures Dr. Stukenborg, Dr. Blackhall, Dr. Harrison, and Dr. Dillon report receiving funding from grants from Centers for Medicare and Medicaid Services Innovation Center during the conduct of the study. Dr. Read reports receiving funding from grants from Centers for Medicare and Medicaid Services Innovation Center during the conduct of the study, and grants from Accuray, outside the submitted work.

Open Access This article is distributed under the terms of the Creative Commons Attribution-NonCommercial 4.0 International License (http://creativecommons.org/licenses/by-nc/4.0/), which permits any noncommercial use, distribution, and reproduction in any medium, provided you give appropriate credit to the original author(s) and the source, provide a link to the Creative Commons license, and indicate if changes were made. 


\section{References}

1. Giesinger JM, Wintner LM, Oberguggenberger AS, Gamper EM, Fiegl M, Denz H, Kemmler G, Zabernigg A, Holzner B (2011) Quality of life trajectory in patients with advanced cancer during the last year of life. J Palliat Med 14:904-912

2. Downey L, Engelberg RA (2010) Quality-of-life trajectories at the end of life: assessments over time by patients with and without cancer. J Am Geriatr Soc 58:472-479

3. Lunney JR, Lynn J, Foley DJ, Lipson S, Guralnik JM (2003) Patterns of functional decline at the end of life. Jama 289:2387-2392

4. Morden NE, Chang CH, Jacobson JO, Berke EM, Bynum JP, Murray KM, Goodman DC (2012) End-of-life care for Medicare beneficiaries with cancer is highly intensive overall and varies widely. Health Aff (Millwood) 31:786-796

5. Bakitas MA, Tosteson TD, Li Z, Lyons KD, Hull JG, Li Z, DionneOdom JN, Frost J, Dragnev KH, Hegel MT, Azuero A, Ahles TA (2015) Early versus delayed initiation of concurrent palliative oncology care: patient outcomes in the ENABLE III randomized controlled trial. J Clin Oncol 33:1438-1445

6. Temel JS, Greer JA, Muzikansky A, Gallagher ER, Admane S, Jackson VA, Dahlin CM, Blinderman CD, Jacobsen J, Pirl WF, Billings JA, Lynch TJ (2010) Early palliative care for patients with metastatic non-small-cell lung cancer. N Engl J Med 363:733-742

7. Connor SR, Pyenson B, Fitch K, Spence C, Iwasaki K (2007) Comparing hospice and nonhospice patient survival among patients who die within a three-year window. J Pain Symptom Manag 33: 238-246

8. Earle CC, Landrum MB, Souza JM, Neville BA, Weeks JC, Ayanian JZ (2008) Aggressiveness of cancer care near the end of life: is it a quality-of-care issue? J Clin Oncol 26:3860-3866

9. Braga S (2011) Why do our patients get chemotherapy until the end of life? Ann Oncol 22(11):2345-2348. doi:10.1093/annonc/mdr416

10. Wright AA, Zhang B, Keating NL, Weeks JC, Prigerson HG (2014) Associations between palliative chemotherapy and adult cancer patients' end of life care and place of death: prospective cohort study. BMJ 348:g1219

11. Saito AM, Landrum MB, Neville BA, Ayanian JZ, Earle CC (2011) The effect on survival of continuing chemotherapy to near death. BMC Palliat Care 10:14

12. Bottomley A, Efficace F (2006) Predicting survival in advanced cancer patients: is it possible with patient-reported health status data? Ann Oncol 17:1037-1038

13. Gotay CC, Kawamoto CT, Bottomley A, Efficace F (2008) The prognostic significance of patient-reported outcomes in cancer clinical trials. J Clin Oncol 26:1355-1363

14. Efficace F, Lo-Coco F (2013) Using patient-reported health status to improve prognostic assessment in patients with acute myeloid leukemia: current challenges and future applications. Haematologica 98:7-9

15. Secord AA, Coleman RL, Havrilesky LJ, Abernethy AP, Samsa GP, Cella D (2015) Patient-reported outcomes as end points and outcome indicators in solid tumours. Nat Rev Clin Oncol 12:358-370

16. Cella D, Yount S, Rothrock N, Gershon R, Cook K, Reeve B, Ader D, Fries JF, Bruce B, Rose M (2007) The Patient-Reported Outcomes Measurement Information System (PROMIS): progress of an NIH Roadmap cooperative group during its first two years. Med Care 45:S3-s11

17. Cella D, Riley W, Stone A, Rothrock N, Reeve B, Yount S, Amtmann D, Bode R, Buysse D, Choi S, Cook K, Devellis R, DeWalt D, Fries JF, Gershon R, Hahn EA, Lai JS, Pilkonis P, Revicki D, Rose M, Weinfurt K, Hays R (2010) The PatientReported Outcomes Measurement Information System (PROMIS) developed and tested its first wave of adult self-reported health outcome item banks: 2005-2008. J Clin Epidemiol 63:1179-1194
18. Wagner LI, Schink J, Bass M, Patel S, Diaz MV, Rothrock N, Pearman T, Gershon R, Penedo FJ, Rosen S, Cella D (2015) Bringing PROMIS to practice: brief and precise symptom screening in ambulatory cancer care. Cancer 121:927-934

19. Garcia SF, Cella D, Clauser SB, Flynn KE, Lad T, Lai JS, Reeve BB, Smith AW, Stone AA, Weinfurt K (2007) Standardizing patient-reported outcomes assessment in cancer clinical trials: a patient-reported outcomes measurement information system initiative. J Clin Oncol 25:5106-5112

20. Stukenborg GJ, Blackhall L, Harrison J, Barclay JS, Dillon P, Davis MA, Mallory M, Read P (2014) Cancer patient-reported outcomes assessment using wireless touch screen tablet computers. Qual Life Res 23:1603-1607

21. Basch E, Abernethy AP, Mullins CD, Reeve BB, Smith ML, Coons SJ, Sloan J, Wenzel K, Chauhan C, Eppard W, Frank ES, Lipscomb J, Raymond SA, Spencer M, Tunis S (2012) Recommendations for incorporating patient-reported outcomes into clinical comparative effectiveness research in adult oncology. J Clin Oncol 30:4249 4255

22. Agency for Healthcare Research and Quality. Clinical Classifications Software (CCS) for ICD-9-CM. Healthcare Cost and Utilization Project (HCUP). March 2015. Agency for Healthcare Research and Quality, Rockville, MD. Accessed on June 30, 2015 at www.hcup-us.ahrq.gov/toolssoftware/ccs/ccs.jsp.

23. PROMIS Scoring Manuals. Patient Reported Outcomes Measurement Information System. Assessment Center. Accessed on June 30, 2015 at http://www.assessmentcenter.net/

24. Liu H, Cella D, Gershon R, Shen J, Morales LS, Riley W, Hays RD (2010) Representativeness of the Patient-Reported Outcomes Measurement Information System Internet panel. J Clin Epidemiol 63:1169-1178

25. Singer DWJ (2003) Applied longitudinal data analysis: modeling change and event occurrence. Oxford University Press, New York

26. Desquilbet L, Mariotti F (2010) Dose-response analyses using restricted cubic spline functions in public health research. Stat Med 29:1037-1057

27. Burnham KP, Anderson DR (2002) Model selection and multimodel inference: a practical information-theoretic approach. Springer-Verlag

28. Therneau TMGP (2000) Modeling survival data: extending the Cox model. Springer-Verlag, New York

29. Allison P (2010) Survival analysis using SAS: a practical guide. SAS Publishing, Cary, NC

30. Buchholz A, Sauerbrei W (2011) Comparison of procedures to assess non-linear and time-varying effects in multivariable models for survival data. Biom J 53:308-331

31. Fisher LD, Lin DY (1999) Time-dependent covariates in the Cox proportional-hazards regression model. Annu Rev Public Health 20:145-157

32. Cleeland CS, Sloan JA, Cella D, Chen C, Dueck AC, Janjan NA, Liepa AM, Mallick R, O’Mara A, Pearson JD, Torigoe Y, Wang XS, Williams LA, Woodruff JF (2013) Recommendations for including multiple symptoms as endpoints in cancer clinical trials: a report from the ASCPRO (Assessing the Symptoms of Cancer Using Patient-Reported Outcomes) Multisymptom Task Force. Cancer 119:411-420

33. Quinten C, Maringwa J, Gotay CC, Martinelli F, Coens C, Reeve BB, Flechtner H, Greimel E, King M, Osoba D, Cleeland C, Ringash J, Schmucker-Von Koch J, Taphoorn MJ, Weis J, Bottomley A (2011) Patient self-reports of symptoms and clinician ratings as predictors of overall cancer survival. J Natl Cancer Inst 103:1851-1858

34. Oncology Care Model. The Center for Medicare and Medicaid Innovation (Innovation Center) at the Centers for Medicare and Medicaid Services (CMS). Accessed on June 30, 2015 at http:// innovation.cms.gov/initiatives/Oncology-Care/ 